\title{
Coincidence of Anterior Communicating Artery Aneurysm in a Patient With Carotid Body Tumor: A Case Report
}

\author{
Afsoun Seddighi, Sima Behrouzian, Amir Nikouei, Amir Saied Seddighi* \\ Shohada Tajrish Comprehensive Neurosurgical Center of Excellence, Functional Neurosurgery Research Center, Shohada \\ Tajrish Hospital, Shahid Beheshti University of Medical Sciences, Tehran, Iran
}

\begin{abstract}
Background: Intracranial aneurysms (IAs) are focal pathologic dilation of cerebral vasculature, which mostly affect the anterior circulation of brain. Carotid body tumors (CBTs) are the most common head and neck parasympathetic paragangliomas. These slow growing neoplasms may cause hypertension along with catecholamine release symptoms, mostly in patients in their fourth decade. This is the second reported case of simultaneous presentation of CBT and IA in a male patient.

Case Presentation: A 54-year-old male with positive history of hypertension presented with isolated acute weakness of right upper extremity. Bilateral Doppler ultrasound of carotid arteries showed a mass at left carotid bifurcation, which was confirmed by vessels computed tomography (CT) angiography. CT scan also demonstrated anterior communicating artery (A-Com) aneurysm. Digital subtraction angiography (DSA) confirmed a right sided A-com artery aneurysm. Aneurismal repair was performed prior to CBT removal.

Conclusion: Although multifactorial etiologies, such as hypertension, atherosclerosis and congenital predisposition with vascular abnormalities exists; this case raises the possibility of etiologic relationship between hypertension and hypertensive crises due to catecholamine release and aneurismal development and rupture. Avoidance of possible life threatening complications of aneurismal rupture necessitates preoperative evaluation for CBT in patients with established diagnosis of IA.

Keywords: Intracranial aneurysm; Carotid body tumor; Anterior communicating artery aneurysm; Hypertension; Screening
\end{abstract}

*Correspondence to

Amir Saied Seddighi, MD,

Department of Neurosurgery,

Shohada Tajrish Hospital,

Tehran, Iran.

Tel: +98(912)21551591;

Email: a_sedighi@sbmu.ac.ir

Published online October 8 , 2017

Citation: Seddighi A, Behrouzian S, Nikouei A, Seddighi AS. Coincidence of anterior communicating artery aneurysm in a patient with carotid body tumor: a case report. Int Clin Neurosci J. 2017 Autumn;4(4):157-159. doi: 10.15171/icnj.2017.07.

\section{Introduction}

Intracranial aneurysms (IAs) are focal pathologic dilation of cerebral arterial lumen as a consequence of congenital or acquired conditions. ${ }^{1}$ Autopsy series and angiographic studies report up to $3.6 \%$ and $6 \%$ prevalence of IA in general population, respectively. ${ }^{2}$ More than $85 \%$ of IAs are found in the anterior circulation, usually in the anterior communicating artery followed by the internal carotid artery (ICA); also, the basilar apex is the most common site for IAs in posterior circulation. ${ }^{3}$ IAs larger than 10 $\mathrm{mm}$ and symptomatic ones, tend to have greater rupture risk, which results in subarachnoid hemorrhage (SAH). Although systolic hypertension remains an independent predictor of $\mathrm{SAH},{ }^{5}$ symptomatic carotid body tumor (CBT) may be responsible for sudden increase in blood pressure. $^{6}$

Paraganglion cells of the carotid body, which originates from neural crest, may become neoplastic. ${ }^{7}$ CBTs which represent more than half of head and neck paragangliomas, tend to occur at carotid bifurcation. ${ }^{8}$ Although CBTs mostly present as slow growing nontender cervical mass, patients may give hypertension fluctuations and palpitations as catecholamine release symptoms. ${ }^{9}$ Association between IA and CBT has been reported in just one case. ${ }^{10}$ Since the role of blood pressure in pathogenesis and formation of IAs has not been definitely cleared, this association could raise the question that whether CBT is responsible for aneurismal formation and further rupture.

We report the second case of anterior communicating artery (A-Com) aneurysm and left CBT coincidence in a 54-year-old male patient.

\section{Case Presentation}

The patient was a 54-year-old male presenting with acute right upper extremity weakness. His medical history revealed hypertension without any crisis or palpitation, flushing and sweating. Physical examination showed 
right hand weakness without any neurological deficits and any palpable cervical mass. Bilateral Doppler ultrasound of carotid arteries showed a solid mass at left carotid bifurcation. Brain and carotid vessels computed tomography (CT) - angiography demonstrated a hypervascular mass at left carotid bifurcation and A-Com artery aneurysm. Further evaluation was performed by digital subtraction angiography (DSA), which revealed CBT with splaying of the ICA and external carotid artery (ECA) at left carotid bifurcation, plus a right sided A-Com artery aneurysm (Figure 1).

Based on clinical and radiographic findings and high risk of aneurismal rupture, CBT removal was postponed after aneurysm repair. Right pterional craniotomy was performed and the aneurysm was clipped with a $7-\mathrm{mm}$ curved and mini clip $(3 \mathrm{~mm})$. Later, patient underwent for his CBT removal, and excised lesion sent for histopathological examination. Round and hyperchromatic nuclei with abundant granular cytoplasm was noted microscopically; also, hematoxylin and eosin (H\&E) staining revealed "zellaballen" pattern, which is characteristic of paragangliomas (Figure 2).

\section{Discussion}

The carotid body is thought to originate from third branchial arch and it derives from neural crest cells, ${ }^{11}$

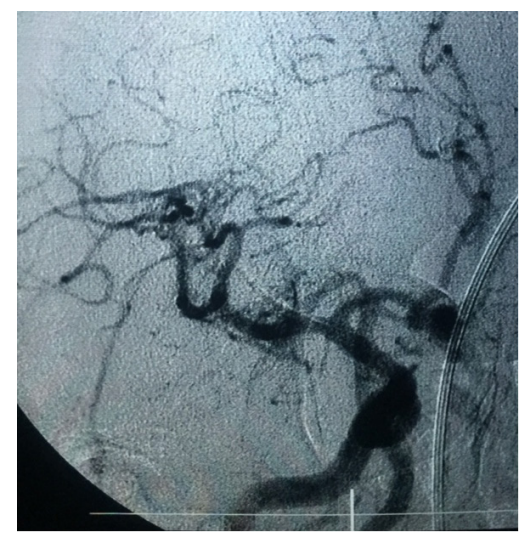

Figure 1. Digital subtraction angiography shows carotid body tumor at left carotid bifurcation and right sided anterior communicating artery aneurysm.

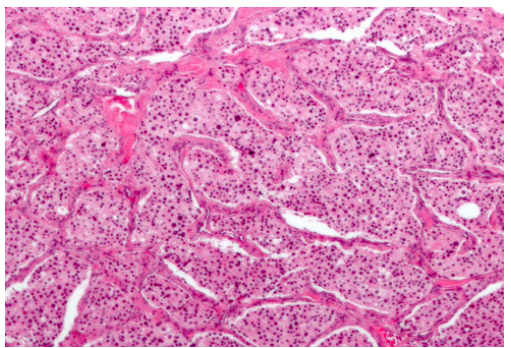

Figure 2. Histopathological features of carotid body tumor including well defined zellaballen pattern, round hyperchromatic nuclei and granular cytoplasm are shown. and also innervated by the vagus and glossopharyngeal nerves and the cervical sympathetic ganglia. ${ }^{12}$ CBTs, also known as chemodectoma, are rare parasympathetic paragangliomas, affecting patients in their mid-40s. ${ }^{13}$ Since CBT mostly present as non tener palpable mass on the neck, lesion such as enlarged lymph node, nerve sheath tumor, branchial cleft cyst, and metastatic carcinoma should be kept in mind. ${ }^{14}$ Our presented case represents the second reported case of simultaneous CBT and IA. Saccular or berry IAs, are congenital lesions which constitute more than $90 \%$ of all IAs. These types of aneurysms usually rupture into subarachnoid space, causing aneurismal SAH which carries more than $60 \%$ mortality rate at initial hemorrhage. ${ }^{15}$ IAs appear as a well-defined round hyper density mass with calcification on CT scan images. Post contrast images may show uniform enhancement; however rim enhancement and filling defect is detected in case of thrombosed aneurysm. DSA remains the gold standard for IA evaluation. ${ }^{16} \mathrm{~A}$ multifactorial etiology is responsible for disruption of internal elastic lamina's integrity. Associated muscular defects of the tunica media and weak support of adjacent parenchyma provide more sensitive structure to hemodynamic stress on the arterial wall. ${ }^{17}$ Controversy exists about the pathophysiologic role of systemic arterial hypertension in the development of IAs; while many have advocated the role of hypertension, ${ }^{18-20}$ some authors have not found such significant relationship. ${ }^{21}$ In addition, it is well known that some hypertensive disorders such as polycystic kidney disease and coarctation of the aorta are frequently associated with IAs. ${ }^{22,23}$ Although IA screening in certain high risk groups such as coarctation of the aorta has been discussed in international guidelines, uncertainty exists about whether hypertension alone has acceptable cost-benefit ratio to prompt IA screening. ${ }^{24}$

\section{Conclusion}

Simultaneous presentation of CBT with IA in our presented case promotes the importance of screening for catecholamine secreting tumors in patients with established diagnosis of IA and previous hypertensive attacks. Identification of such lesions in mentioned patient requires careful blood pressure monitoring to avoid life threatening complication of aneurismal rupture.

\section{Conflict of Interest Disclosures}

The authors declare that they have no conflict of interests.

Ethical Statement

Written informed consent was obtained from the patient with ethical approval of Ethics Committee of Shohada Tajrish hospital.

\section{References}

1. Gasparotti R, Liserre R. Intracranial aneurysms. Eur Radiol. 2005;15:441-7.

2. Rinkel GJ, Djibuti M, Algra A, van Gijn J. Prevalence and risk of rupture of intracranial aneurysms: a systematic review. Stroke. 1998;29:251-6. 
3. Bonneville F, Sourour N, Biondi A. Intracranial aneurysms: an overview. Neuroimaging Clin N Am. 2006;16(3):371-82.

4. Juvela S, Porras M, Poussa K. Natural history of unruptured aneurysms. J Neurosurg. 2000;93:379-387.

5. Juvela S. Prehemorrhage risk factors for fatal intracranial aneurysm rupture. Stroke. 2003;34:1852-8.

6. de Franciscis S, Grande R, Butrico L, Buffone G, Gallelli L, Scarcello E, et al. Resection of carotid body tumors reduces arterial blood pressure. An underestimated neuroendocrine syndrome. Int J Surg. 2014;12:S63-7.

7. Davidovic LB, Djukic VB, Vasic DM, Sindjelic RP, Duvnjak $\mathrm{SN}$. Diagnosis and treatment of carotid body paraganglioma: 21 years of experience at a clinical center of Serbia. World J Surg Oncol. 2005;3(1):10.

8. Georgiadis GS, Lazarides MK, Tsalkidis A, Argyropoulou P, Giatromanolaki A. Carotid body tumor in a 13-year-old child: Case report and review of the literature. I Vasc Surg. 2008;47(4):874-880.

9. Milewski C. Morphology and clinical aspects of paragangliomas in the area of head-neck. HNO 1993;41:526531.

10. Inci S, Bertan V. Catecholamine-secreting carotid body tumor and intracranial aneurysm: coincidence? Surg Neurol. 2000;53(5):488-92.

11. Meyer FB, Sundt TM, Pearson BW. Carotid body tumors: a subject review and suggested surgical approach. J Neurosurg. 1986;64:377-85.

12. Eyzaguirre C, Zapata P. Perspectives in carotid body research J App Physiol. 1984;57:931-57.

13. Sevilla Garcia MA, Llorente Pendas JL, Rodrigo Tapia JP, Garcia Rostan G, Suarez Fente V, Coca Pelaz A, et al. [Head and neck paragangliomas: revision of 89 cases in 73 patients] Acta Otorrinolaringol Esp. 2007;58(3):94-100.

14. Anand VK, Alemar GD, Sanders TS. Management of the internal carotid artery during carotid body tumor surgery.
Laryngoscope. 1979;105:231-5.

15. Weir B, Disney L, Karrison T. Sizes of ruptured and unruptured aneurysms in relation to their sites and the ages of patients. J Neurosurg. 2002;96:64-70.

16. Papke K, Kuhl CK, Fruth M, Haupt C, Schlunz-Hendann M, Sauner D, et al. Intracranial aneurysms: role of multidetector CT angiography in diagnosis and endovascular therapy planning. Radiology. 2007;244(2):532-40.

17. Frösen J, Tulamo R, Paetau A, Laaksamo E, Korja M, Laakso $A$, et al. Saccular intracranial aneurysm: pathology and mechanisms. Acta Neuropathol. 2012 Jun;123(6):773-86.

18. Gu YX, Chen XC, Song DL, Leng B, Zhao F. Risk factors for intracranial aneurysm in a Chinese ethnic population. Chin Med J (Engl). 2006;119(16):1359-64.

19. de la Monte SM, Moore GW, Monk MA, Hutchins GM. Risk factors for the development and rupture of intracranial berry aneurysms. Am J Med. 1985;78(6 Pt 1):957-64.

20. Taylor CL, Yuan Z, Selman WR, Ratcheson RA, Rimm AA. Cerebral arterial aneurysm formation and rupture in 20,767 elderly patients: hypertension and other risk factors. J Neurosurg. 1995;83(5):812-9.

21. Andrew RJ, Spiegel PK. Intracranial aneurysms. Age, sex, blood pressure, and multiplicity in an unselected series of patients. J Neurosurg. 1979;51:27-32.

22. Fehlings MG, Gentili $F$. The association between polycystic kidney disease and cerebral aneurysms. Can J Neurol Sci. 1991;18:505-9.

23. Fukuda H, Sako K, Yonemasu Y. Coarctation of the descending aorta with aneurysm of the anterior communicating artery. Surg Neurol .1985;23:380 -2.

24. Rodrigues JC, Hassan N, Williams M, Burchell AE, Ratcliffe $L E$, Hamilton $M$, et al. Should we screen for intracranial aneurysms (IAs) in systemic hypertension at the time of cardiac magnetic resonance (CMR)? J Cardiovasc Magn Reson. 2015;17(suppl 1):411. 